\section{Helminthen-Proteine: Allergieprotektoren der Zukunft?}

Gastrointestinal parasitierende Fadenwürmer (Nematoda) können immunmodulatorische Effekte entfalten, die sich therapeutisch unter anderem bei Autoimmunerkrankungen und Allergien nutzen lassen. Patientenfeundlicher wäre sicher die Applikation der wirksamen Inhaltsstoffe dieser Würmer.

\begin{abstract}
n Tiermodellen und Experimentalstudien mit autoimmun oder allergisch erkrankten Patienten ist die immunmodulatorische Wirksamkeit einer Infektion mit intestinal lebenden Helminthen nachgewiesen. Die Verwendung von Eiern des Schweinepeitschenwurms (Trichuris suis ova) als immunmodulatorische Therapie z.B. bei chronisch entzündlichen Darmerkrankungen befindet sich bereits in der klinischen Entwicklung und könnte bald zugelassen werden. Denkbar wäre auch die Anwendung der wirksamkeitstragenden Proteine - doch hier fehlt es noch an Wissen.

Eine Berliner Arbeitsgruppe charakterisierte deshalb zunächst die wichtigsten exkretorischen bzw. sekretorischen Proteine (E/S-Proteine) von Trichuris suis im ersten Larvenstadium - der ver-
\end{abstract}

mutlich für die immunologischen Anpassungsmechanismen bedeutendsten Entwicklungsphase. Dann analysierten sie die immunsuppressiven Effekte für allergische Atemwegserkrankungen in vitro und in einem Mausmodell.

Wurden die E/S-Proteine in vivo während der allergischen Sensibilisierung der Mäuse gegeben, ließen sich Atemwegshyperreaktivität, die Einwanderung von Entzündungszellen in die Bronchien und die allergenspezifische IgE-Produktion unterdrücken. Drei der immunologisch aktiven Wurmlarven-Proteine konnten identifiziert werden. Möglicherweise ist ein Interleukin(IL)-10-abhängiger Wirkmechanismus an der beobachteten Wirkung beteiligt. IL-10 ist ein wichtiges immunregulatorisches Zytokin.

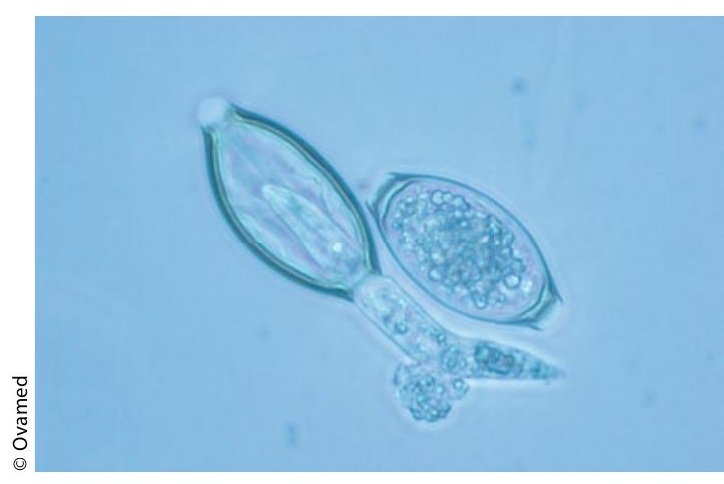

Ein Schweinepeitschenwurm (Trichuris suis) schlüpft aus dem Ei.

Fazit: Proteine, die von Schweinepeitschenwurm-Larven abgesondert werden, zeigen im Mausmodel protektive Effekte gegen eine allergische Atemwegsallergie. Möglicherweise wirken die Eiweißstoffe unter anderem über einen IL-10-abhängigen Mechanismus immunmodulatorisch.

Dr. Barbara Kreutzkamp

Ebner $\mathrm{F}$ et al. Therapeutic potential of larval excretory/secretory proteins of the pig whipworm Trichuris suis in allergic disease. Allergy 2014;69:1489-97

\section{Seltener Atemwegsinfekte dank roher Kuhmilch?}

Auf vielen Bauernhöfen ist es noch üblich, Säuglinge mit roher Kuhmilch zu ernähren. Dies hat die PASTURE-Studiengruppe genutzt, um zu prüfen, wie sich unbehandelte Kuhmilch auf Atemwegsinfektionen und Rhinitis auswirkt.

D ie PASTURE-Studie (PASTURE, Protection against Allergy - Study in Rural Environments) ist eine internationale Kohortenstudie mit fast 1.000 Säuglingen aus ländlichen Gebieten in Österreich, Finnland, Frankreich, Bayern und der Schweiz. Wöchentlich mussten die Eltern festhalten, ob die Säuglinge mit unbehandelter Rohmilch, erwärmter, pasteurisierter oder ultrahocherhitzter Milch (H-Milch) ernährt wurden. Zudem sollten sie Angaben über Rhinitis, Atemwegsinfektionen, Otitis media und Fieber machen. Nach zwölf Monaten wurde der Entzündungsparameter C-reaktives Protein (CRP) im Blut gemessen.

Anfangs wurden etwa $80 \%$ der Kinder gestillt, bis zum Ende des Untersuchungs- zeitraums waren es nur noch $20 \%$. Mit etwa drei Monaten wurde begonnen, die Säuglinge mit Kuhmilch zu ernähren. Im Alter von zwölf Monaten tranken je $20 \%$ der Kinder regelmäßig gekochte oder pasteurisierte Kuhmilch und je $12 \%$ H-Milch oder Rohmilch.

Die Auswertung der Daten ergab, dass das Trinken von roher Kuhmilch im Vergleich zu H-Milch die Wahrscheinlichkeit für Atemwegsinfektionen um $23 \%$ (Odds Ratio [OR]: 0,77; 95\%-Konfidenzintervall: 0,59-0,99) und für Mittelohrentzündung um $86 \%$ (95\%-Konfidenzintervall: $0,05-0,42$ ) verringert. Auch das Risiko für Rhinitis und Fieber wurde deutlich gesenkt (29\% bzw. $31 \%$ ). Schließlich hatten Kinder, die mit roher Kuhmilch ernährt worden waren, im
Alter von einem Jahr signifikant niedrigere CRP-Werte als die mit den anderen Milchsorten ernährten Kinder. Erstautor Georg Loss, München, folgert: „Der Konsum von Rohmilch könnte also das Risiko senken, später an Asthma zu erkranken."

Fazit: Der Genuss von Rohmilch ist mit einem deutlich niedrigeren Risiko für Atemwegsinfekte und Fieber sowie Otitis media verbunden. Im Vergleich zur H-Milch sinkt das Erkrankungsrisiko um bis zu $30 \%$. Die Wissenschaftler plädieren daher dafür, neue, die Milchkomponenten schonendere industrielle Verfahren zu entwickeln, um die schützende Wirkung der Kuhmilch zu bewahren.

Peter Leiner

Loss $\mathrm{G}$ et al. Consumption of unprocessed cow's milk protects infants from common respiratory infections. J Allergy Clin Immunol 2014; online 19. Oktober 